\title{
Amyloid tumor of the stomach simulating an obstructing gastric carcinoma: case report and review of the literature
}

Systemic amyloidosis is known to rarely affect the stomach [1-5]. A 59-year-old male patient presented with intermittent nausea, vomiting, daily bowel movements, and weight loss of $20 \mathrm{~kg}$ over 2 months. His past medical history was significant for arterial hypertension, prostate cancer for 10 years, multiple myeloma, and recent renal failure. Barium upper gastrointestinal X-ray series revealed a large constricting lesion in the gastric antrum and proximal duodenum suggestive of malignancy ( $\bullet$ Fig. 1). Upper gastrointestinal endoscopy revealed a large, fungating, friable mass which obstructed nearly $75 \%$ of the prepyloric lumen ( Fig. 2). Pathological analysis of the biopsies revealed amyloid deposits on the muscularis mucosae.

Exploratory laparotomy found a $4 \times 5 \mathrm{~cm}$ obstructing soft mass in the prepyloric antrum. A Billroth II partial gastrectomy was performed. Pathological analysis showed infiltration of the muscularis mucosae and muscularis propria by amyloid and no malignancy. The postoperative course was marked by progressive impairment of the gastrointestinal motility. The patient died 5 months later.

Gastric outlet obstruction by an amyloid tumor is exceptional, with five published reports ( Table 1). All patients were treated with surgery, a most reliable management of gastric outlet obstruction. In patients with known systemic amyloidosis who present with gastric outlet obstruction, regional amyloid deposition should be included in the differential diagnosis, along with other, more common causes such as ulcer or carcinoma. The obstruction can co-exist with a previously unrecognized amyloid gastrointestinal motility disorder, which can worsen the outcome.

\section{Acknowledgments \\ $\nabla$}

This material is the result of work supported with resources and use of facilities at the John D. Dingell VAMC, Detroit, Michigan, USA.

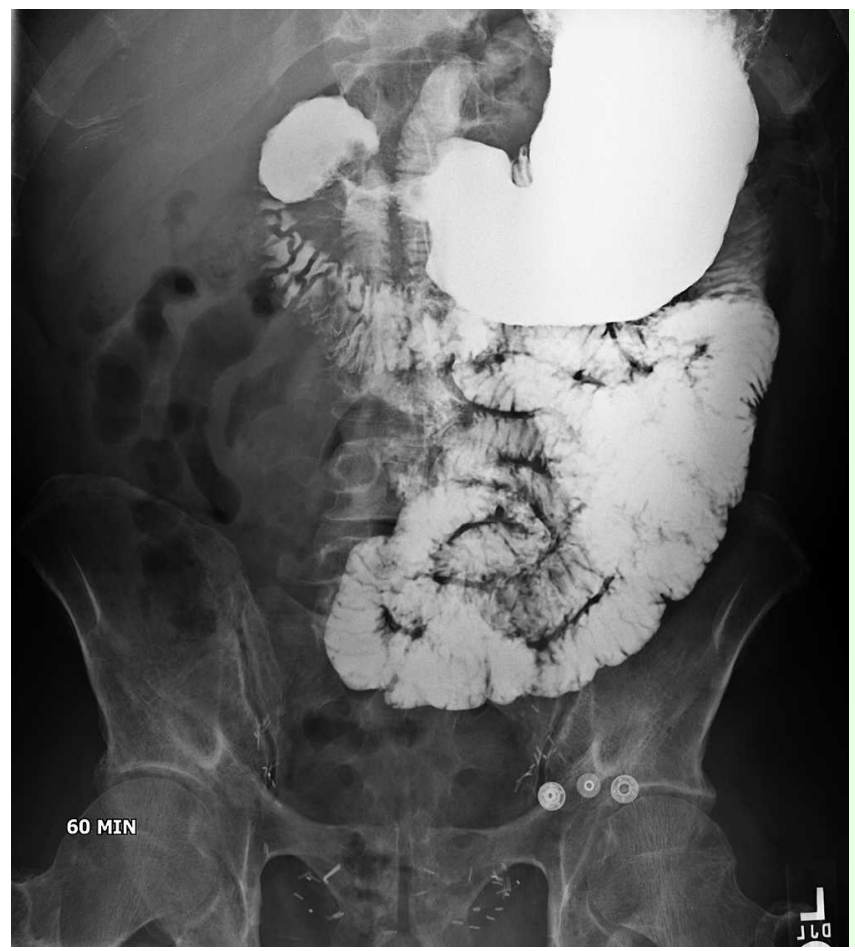

Fig. 1 Barium meal showing a constricting lesion of the gastric antrum and proximal duodenum suggestive of gastric cancer.

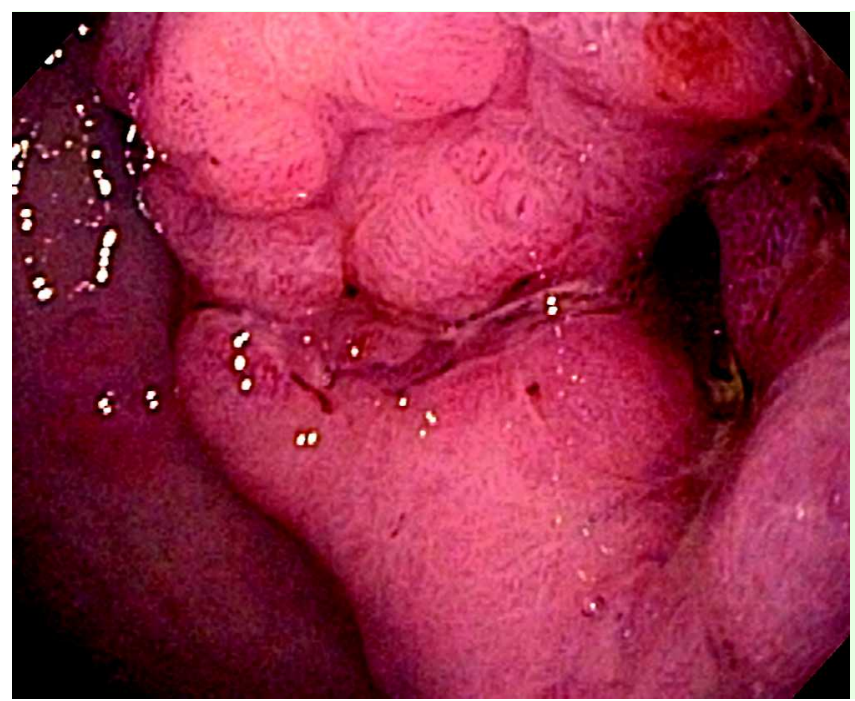

Fig. 2 Endoscopic appearance of a large circumferential, fungating, friable mass in the prepyloric antrum. 


\begin{tabular}{|c|c|c|c|c|c|c|c|}
\hline Study & $\begin{array}{l}\text { Age/ } \\
\text { gender }\end{array}$ & $\begin{array}{l}\text { Underlying } \\
\text { disorder }\end{array}$ & $\begin{array}{l}\text { Duration of } \\
\text { GI disorder }\end{array}$ & Diagnosis & Treatment & $\begin{array}{l}\text { Outcome/ } \\
\text { cause of death }\end{array}$ & $\begin{array}{l}\text { Table } 1 \text { Previously published } \\
\text { cases of gastric amyloid tumor }\end{array}$ \\
\hline Golden [1] & $66 / F$ & $\begin{array}{l}\text { Primary } \\
\text { amyloidosis }\end{array}$ & 16 years & $\begin{array}{l}\text { Surgical } \\
\text { specimen }\end{array}$ & $\begin{array}{l}\text { Partial } \\
\text { gastrectomy }\end{array}$ & $\begin{array}{l}\text { Died from malnutri- } \\
\text { tion ( } 69 \text { days) }\end{array}$ & $\begin{array}{l}\text { causing gastric outlet obstruc- } \\
\text { tion. }\end{array}$ \\
\hline $\begin{array}{l}\text { Shnider and } \\
\text { Burka [2] }\end{array}$ & $52 / F$ & $\begin{array}{l}\text { Multiple } \\
\text { myeloma }\end{array}$ & 6 months & $\begin{array}{l}\text { Surgical } \\
\text { specimen }\end{array}$ & $\begin{array}{l}\text { Partial } \\
\text { gastrectomy }\end{array}$ & $\begin{array}{l}\text { Died from anastomo- } \\
\text { tic leak ( } 15 \text { days) }\end{array}$ & \\
\hline $\begin{array}{l}\text { Klingen- } \\
\text { berg [3] }\end{array}$ & $63 / \mathrm{M}$ & $\begin{array}{l}\text { Primary } \\
\text { amyloidosis }\end{array}$ & 1.5 years & $\begin{array}{l}\text { Surgical } \\
\text { specimen }\end{array}$ & $\begin{array}{l}\text { Partial } \\
\text { gastrectomy }\end{array}$ & $\begin{array}{l}\text { Died from pulmonary } \\
\text { embolism ( } 5 \text { days) }\end{array}$ & \\
\hline Lewis [4] & $68 / F$ & $\begin{array}{l}\text { Multiple } \\
\text { myeloma }\end{array}$ & months** & Endoscopy & $\begin{array}{l}\text { Gastrojeju- } \\
\text { nostomy }\end{array}$ & $\begin{array}{l}\text { Died from Gl bleeding } \\
\text { ( } 3 \text { months) }\end{array}$ & \\
\hline $\begin{array}{l}\text { Cryer and } \\
\text { Kissane [5] }\end{array}$ & $37 / F$ & $\begin{array}{l}\text { Multiple } \\
\text { myeloma }\end{array}$ & 1 year & $\begin{array}{l}\text { Surgical } \\
\text { specimen }\end{array}$ & $\begin{array}{l}\text { Gastrojeju- } \\
\text { nostomy }\end{array}$ & Not reported & \\
\hline
\end{tabular}

* I. e., days after surgery; Gl, gastrointestinal.

${ }^{* *}$ the authors did not specify how many days after surgery.

J. E. Losanoff ${ }^{1}$, F. Antaki ${ }^{2}$, W. A. Salwen ${ }^{1}$, D. Edelman ${ }^{1}$, A. Reddy ${ }^{2}$, E. Levi ${ }^{3}$,

M. D. Basson ${ }^{1}$

1 Department of Surgery, John D. Dingell VAMC and Wayne State University,

Detroit, Michigan, USA

2 Department of Medicine, Division of Gastroenterology, John D. Dingell VAMC and Wayne State University, Detroit, Michigan, USA

3 Department of Pathology, John D. Dingell VAMC and Wayne State University, Detroit, Michigan, USA

\section{References}

1 Golden A. Primary systemic amyloidosis of the alimentary tract. Arch Int Med 1945; 75: $413-416$

2 Shnider B, Burka P. Amyloid disease of the stomach simulating gastric carcinoma. Gastroenterology 1955; 28: 424-430

3 Klingenberg PH. Amyloidosis of gastrointestinal tract simulating gastric carcinoma. Am J Surg 1958; 96: 713-715

4 Lewis M. Gastrocamera findings in a patient with gastric amyloidosis and multiple myeloma. Gastrointest Endosc 1968; 14: 190 193

5 Cryer PE, Kissane JM. Gastric outlet obstruction in a patient with multiple myeloma. Am J Med 1980; 68: 141 - 148
Bibliography

DOI $10.1055 / \mathrm{s}-0028-1119470$

Endoscopy 2009; 41: E45 - E46

(c) Georg Thieme Verlag KG Stuttgart · New York . ISSN 0013-726X

Corresponding author

\section{J. E. Losanoff, MD}

Department of Surgery (11S)

John D. Dingell VA Medical Center 4646 John R

Detroit

MI 48201

USA

Fax: +1-313-5761002

jelosanoff@yahoo.com 